\title{
Cuidado ético ao paciente ostomizado: uma reflexão a partir do Programa Nacional de Segurança do Paciente
}

\author{
Ethical care for ostomy patients: a reflection based on the National Patient Safety Program \\ Atención ética para pacientes con ostomía: una reflexión basada en el Programa Nacional \\ de Seguridad del Paciente
}

Prisciane Cardoso Silva ${ }^{1 *}$, Lisa Antunes Carvalho1, Marina Soares Mota², Edison Luiz Devos Barlem¹, Juliane Portella Ribeiro², Evelyn de Castro Roballo², Rochele Maria Zugno.

\section{RESUMO}

Objetivo: Discutir a ética no cuidado ao paciente estomizado sob a perspectiva do Programa Nacional de Segurança do Paciente. Revisão bibliográfica: A pessoa estomizada enfrenta inúmeras dificuldades como complicações com a estomia, restrições sociais, preocupações com a vida social e insegurança. Compete ao enfermeiro acompanhar a recuperação e a adaptação fisiológica desses pacientes, bem como incentivar a retomada das atividades de vida diária, com qualidade de vida. Para isso, o Programa Nacional de Segurança do Paciente embasa o cuidado seguro a partir de seus objetivos, constituindo um cuidado ético. Assim, o trabalho em enfermagem deve permear os diferentes aspectos da vida das pessoas submetidas à estomia por meio de um cuidado seguro, seguindo as premissas do Programa Nacional de Segurança do Paciente. Considerações finais: Entende-se que o Programa Nacional de Segurança do Paciente com seus eixos, elementos e pressupostos permite a elaboração de um cuidado seguro. A presente reflexão pode contemplar de modo satisfatório o que se entende por cuidado ético.

Palavras-chave: Segurança do paciente, Ética, Estomia, Cuidado de enfermagem.

\begin{abstract}
Objective: To discuss ethics in care for ostomized patients from the perspective of the National Patient Safety Program. Bibliographic review: The ostomized person faces numerous difficulties such as complications with ostomy, social restrictions, concerns about social life and insecurity. It is up to the nurse to monitor the recovery and physiological adaptation of these patients, as well as encourage the resumption of activities of daily living, with quality of care. life. For this, the National Patient Safety Program supports safe care based on its objectives, constituting ethical care. Thus, nursing work must permeate the different aspects of the life of people submitted to ostomy through safe care, following the premises of the National Patient Safety Program. Final considerations: It is understood that the National Patient Safety Program with its axes, elements and assumptions allows the development of safe care. This reflection can satisfactorily contemplate what is meant by ethical care.
\end{abstract}

Keywords: Patient safety, Ethics, Ostomy, Nursing care.

\section{RESUMEN}

Objetivo: discutir la ética en la atención de pacientes ostomizados desde la perspectiva del Programa Nacional de Seguridad del Paciente. Revisión bibliográfica: La persona ostomizada se enfrenta a numerosas dificultades como complicaciones con la ostomía, restricciones sociales, preocupaciones sobre

1 Universidade Federal do Rio Grande (FURG), Rio Grande - RS. *E-mail: priscianecardososilva@gmail.com

2 Universidade Federal de Pelotas (UFPel), Pelotas - RS. 
la vida social y la inseguridad. Corresponde a la enfermera vigilar la recuperación y adaptación fisiológica de estos pacientes, así como incentivar la reanudación de las actividades de la vida diaria, con calidad de vida. Para ello, el Programa Nacional de Seguridad del Paciente apoya la atención segura en base a sus objetivos, constituyendo un cuidado ético. Así, el trabajo de enfermería debe permear los diferentes aspectos de la vida de las personas sometidas a ostomía a través de una atención segura, siguiendo las premisas del Programa Nacional de Seguridad del Paciente. Consideraciones finales: Se entiende que el Programa Nacional de Seguridad del Paciente con sus ejes, elementos y supuestos permite el desarrollo de una atención segura. Esta reflexión permite contemplar satisfactoriamente lo que se entiende por cuidado ético.

Palabras clave: Seguridad del paciente, Ética, Ostomía, Atención de enfermería.

\section{INTRODUÇÃO}

Por definição, a pessoa estomizada é aquela que possui uma abertura artificial dos órgãos internos para o meio externo em decorrência de um procedimento cirúrgico (BRASIL, 2009). Mais especificamente, estoma intestinal refere-se à exteriorização de uma porção intestinal por uma abertura na cavidade abdominal que, dependendo do segmento exposto, terá diferentes nomenclaturas, como colostomia ou ileostomia, podendo ser permanente ou temporária (ANG SG, et al., 2013)

Esse procedimento apresenta elevados índices de morbimortalidade que contribuem para a diminuição da qualidade de vida (QV) das pessoas com estomas, especialmente no que se refere ao bem-estar físico, criando uma preocupação com o estoma, gases e odores, com a eliminação fecal e o vazamento frequente, além de comumente haver diversas complicações com a estomia, como dermatite, hérnia e prolapso, resultando em grande desconforto físico para a pessoa (NÂSVALL P, et al., 2017). Assim, a rotina diária precisa ser reajustada, para que o paciente se adapte da melhor forma à nova realidade. Ainda que seja um recurso terapêutico, a experiência das pessoas com a estomização é constrangedora e complexa e traz inúmeras dificuldades à rotina diária e ao convívio com outras pessoas que precisam ser superadas para que se chegue à adaptação à estomia com QV (MOTA MS, GOMES GC, et al., 2016).

$O$ cuidado integral aos pacientes bem como orientações as famílias devem ser consideradas para a identificação dos sentimentos e dificuldades apresentadas pelo paciente durante a adaptação à nova realidade vivida (SILVA RA, et al., 2020). Considerando que o profissional de Enfermagem desempenha suas atividades com autonomia, baseado em preceitos éticos e legais, técnico-científico e teórico-filosófico, exercendo-as com competência a fim de promover o ser humano na sua integralidade, de acordo com os Princípios da Ética e da Bioética, a assistência às pessoas com estomia deve direcionar seu olhar para questões que vão além dos aspectos físicos, biológicos e psicológicos e refletir acerca do cuidado ético que respeita, compreende e busca conhecer o fenômeno de ser e estar estomizado (COFEN, 2017; MOTA MS, GOMES GC, et al., 2016).

Para isso, conta-se com o Programa Nacional de Segurança do Paciente (PNSP), instituído no ano de 2013 com o objetivo de favorecer a qualificação do cuidado em saúde em todos os estabelecimentos de saúde do território nacional, dando sustentação para o direcionamento do cuidado de enfermagem ao paciente estomizado baseado em princípios éticos. É de fundamental importância alinharmos os pressupostos contidos nesse programa, que trata dentre vários aspectos do cuidado seguro, enquanto um cuidado ético que deve respeitar e valorizar o paciente estomizado na sua fragilidade e necessidade de equilíbrio em sua forma de ser e estar nesse contexto (BRASIL, 2013).

Justifica-se a presente reflexão pois a enfermagem pode e deve pautar-se em um exercício de cuidado seguro, livre de danos e que garanta a integridade física, psicológica e moral dos indivíduos estomizados em diferentes contextos e ao fazê-lo acredita-se exercer também um cuidado ético. Diante disso, emergem reflexões iniciais, como: quais os elementos do PNSP que apontam para um cuidado ético e como ele pode direcionar para uma reflexão ética na prática profissional aos estomizados? Portanto, objetivou-se discutir a ética no exercício do cuidado ao paciente estomizado sob a perspectiva do PNSP. 


\section{REVISÃO BIBLIOGRÁFICA}

\section{Características e Fragilidades das pessoas estomizadas}

A necessidade da construção de um estoma intestinal advém de diversas causas que acometem o sistema digestório, sendo de origem patológica ou por causas externas. As mais frequentes são os traumatismos, as doenças inflamatórias, os tumores e o câncer do intestino (MACÊDO MS, NOGUEIRA LT e LUZ MHBA, 2005). Sendo assim, o paciente estomizado não apenas precisa lidar com a nova vida após o estoma, mas também com a razão pela qual precisou realizar o procedimento e necessita de profissionais preparados técnica e eticamente, para ajudá-lo a lidar com a nova situação (MOTA MS, GOMES GC, et al., 2016).

Dessa forma, surgem dificuldades no retorno à vida cotidiana. Ribeiro WA, et al (2019) diz que, pela incontinência intestinal, a pessoa necessita de um equipamento coletor acoplado ao estoma. Isso leva às preocupações com gases, odores, eliminação fecal e vazamentos, além de complicações como dermatite, hérnia e prolapso, resultando em grande desconforto físico (NÂSVALL P, et al, 2017). Em decorrência disso, as pessoas se deparam com inúmeras restrições sociais ao tentar recuperar papéis e funções sociais anteriores (GANJALIKHANI MK, et al., 2019). As mudanças são tão significativas para essas pessoas que Maciel DBV (2018) argumenta que elas provavelmente não conseguirão voltar às ocupações anteriores e precisarão se readequar a novas funções.

Nesse processo, compete ao enfermeiro acompanhar a recuperação e a adaptação fisiológica desses pacientes, incentivá-los para retornarem as atividades de vida diária com qualidade. Uma consulta de enfermagem com uma abordagem sistematizada, orientações de educação pré-operatórias e um pósoperatório planejado junto com a família são a base para uma boa adaptação da pessoa com estomia intestinal (SAILER M, 2019). Considera-se que o enfermeiro participa de todas essas fases, o que o torna um profissional essencial para um cuidado fundamentado nas boas práticas baseadas em evidências (MOTA MS, GOMES GC, et al., 2016).

Alguns estudos abordam os cuidados direcionados aos aspectos físicos com pouco aprofundamento quanto aos aspectos psicossociais dos pacientes estomizados. Marques GS et al. (2016) mencionam que os elementos emocionais revelam uma história que transcende o procedimento cirúrgico o que expõe 0 aspecto debilitador de modo mais agudo. Tal fato contribui para a compreensão de que os pacientes ostomizados não se resumem as questões biológicas e físicas, de adaptação ou reabilitação, mas sim, levanos a refletir sobre como os limites impostos podem afetá-los em suas emoções e aspectos sociais, que se fragilizados, potencializam a baixa autoestima, o que afeta a satisfação e prazer na vida e seu cotidiano (REPIĆ G, et al., 2018).

Com isso, percebe-se que a experiência vivida pelas pessoas com estomia é um processo doloroso, que acompanha medo, limitações, constrangimento, incômodo e dúvidas, fazendo com que haja a necessidade de atenção nos mais diversos âmbitos da vida. Essas pessoas precisam retomar sua autonomia, ressignificar sua identidade e reconfigurar seu papel social. Os profissionais de enfermagem precisam estar aptos para auxiliá-las nesse processo, estando munidos de conhecimento, a fim de desenvolver 0 autocuidado e ajudá-las a visualizar as potencialidades que ainda permanecem. (SAILER M, 2019; MOTA MS, GOMES GC, et al., 2016).

\section{Cuidado seguro sob a perspectiva do Programa Nacional de Segurança do Paciente}

O cuidado seguro está permeado da cultura de segurança que deve ser construída nas relações profissionais no interior das organizações de saúde. Envolve além dos aspectos técnicos em relação as ações de saúde especificas, posicionamentos profissionais e percepções da equipe de saúde no que se refere aos padrões de segurança. Construir uma cultura de segurança torna-se um desafio, pois fundamenta-se em mudanças de atitudes profissionais que promovam o bem-estar do paciente, salvaguardando a qualidade da assistência prestada (DE CARVALHO BARBOSA, CAVALCANTE AK, et al., 2015). 
Para que seja possível a construção de um cuidado seguro, os profissionais devem desenvolver competências necessárias para que este cuidado seja efetivo. Assim, competência relaciona-se a uma combinação de Conhecimento, Habilidade e Atitude, apreendida durante processos educativos e expressa pelo indivíduo em comportamentos num determinado contexto profissional. Com isso, os profissionais assumem uma postura proativa, interagindo com os vários setores da instituição e discutindo possíveis soluções para os desafios que encontrarão (PERES AM, et al, 2017; ANVISA, 2014).

A questão posta nos remete a refletir sobre a formação ética dos profissionais, pois sabe-se que esta ocorre principalmente no âmbito social de cada indivíduo e se configura como uma construção diária e as instituições sociais, principalmente a família e a religião, quando presentes, contribuem fortemente na constituição do caráter de cada um. O cuidado seguro sob o olhar do PNSP configura um cuidado ético? Acredita-se que sim, tendo em vista as características que o cuidado seguro sob a perspectiva do PNSP traz consigo. É indissociável pensar em segurança do paciente sem observar componentes éticos que permeiam a assistência de enfermagem neste contexto (MARINS JB, et al., 2018).

Antes mesmo de realizarmos o cuidado, esse constitui-se uma atitude. Essa atitude precisa levar em conta vários aspectos desse paciente em situação de fragilidade diante da nova situação de ser e estar no mundo e de se relacionar com os demais. $\mathrm{Na}$ ausência dessa atitude, a qual exprime a consideração pelo outro e pelas suas necessidades, as ações de cuidar perdem sua motivação e sentido ético, pois deterioram-se e se desvalorizam. Nesse sentido, o cuidado só se revela como ético, quando está alicerçado no reconhecimento da alteridade do outro (MAIA MS, 2009).

Este modo de cuidar pressupõe respeito ao corpo do paciente, consideração sobre seus direitos, limites, potencialidades e reconhecimento das fragilidades frente ao ser e estar estomizado, momento que tanto paciente como profissional devem refletir sobre sua importância ao produzir cuidado. Nesse cenário, 0 PNSP orienta a formação de Núcleos de Segurança do Paciente nas instituições de saúde, os quais objetivam proporcionar e estimular a realização de iniciativas nos serviços, direcionadas à segurança do paciente (BRASIL, 2014).

Assim, além de promover o envolvimento entre pacientes, cuidadores e familiares no processo, permite a ampliação do acesso e da participação e da sociedade nas questões relacionadas à segurança do paciente, por meio do incentivo à disseminação de conteúdos relativos ao tema, bem como da inclusão do mesmo na formação de profissionais da área da saúde. Nessa perspectiva, o PNPS compreende quatro pilares: 0 incentivo a uma assistência segura; a participação e colaboração do indivíduo nas questões que envolvem sua segurança; a inserção da temática da segurança na formação e no ensino; e o fortalecimento da pesquisa que trate das questões da cultura de segurança (BRASIL, 2014).

\section{Cuidado seguro e ético sob a perspectiva do Programa Nacional de Segurança do Paciente}

A ética é a possibilidade de refletir sobre a vida moral, de subsidiar-se de critérios para tomar decisões acerca do que é ou não aceitável, é a capacidade de refletir sobre si mesmo, de questionar o que pode e o que não pode ser realizado, pressupõe liberdade de opção, a consideração do outro, o respeito aos direitos individuais e coletivos (DA SILVEIRA RS, et al., 2014). Considera-se, neste contexto, o cuidado seguro preconizado pelo PNSP como um cuidado ético que os profissionais de enfermagem realizam aos pacientes estomizados, pois eles sofrem além da perda de um pedaço de seu corpo, a modificação de sua imagem, autoestima, autopercepção e absorvem as opiniões e comportamentos alheios quanto à sua condição de estomizado (MOTA MS, GOMES GC, et al., 2016; BRASIL, 2013).

Pode-se inferir que o cuidado seguro inserido nos objetivos do PNSP, constitui-se um cuidado ético, pois possibilita a mudança de paradigma e de comportamento dos profissionais sobre aspectos técnicos e relacionais, instigam a corresponsabilização dos pacientes sobre sua saúde, permitindo que esses se reconheçam como parte e protagonistas desse processo. Também instiga o ensino sobre os temas que envolvem o PNSP nos diferentes níveis, permitindo uma articulação entre teoria e prática, promovendo um novo olhar sobre o cuidado de enfermagem e ampliando a difusão do conhecimento acerca da segurança do paciente na sociedade (BRASIL, 2013). 
Considera-se que o PNSP também possibilita a correção dos eventuais erros profissionais resultantes da sobrecarga de trabalho ou esgotamento profissional, permitindo um repensar acerca das boas práticas, e da forma como se tem exercido o cuidado. A não reflexão sobre esse processo, constitui-se um cuidado antiético, pois desrespeita a individualidade e transgride os aspectos éticos da dimensão do cuidado, dentre eles, um cuidado livre de danos de qualquer natureza (MAIA MS, 2009; BRASIL, 2013; COFEN,2017).

Alinha-se a esta ideia, o cuidado ético que pressupõe a somatória das ações técnicas em saúde, ou seja, cuidado ao estomizado e a suas necessidades, com a atitude ética que reforça um fazer com mínimo de dano ou riscos ao paciente. Pode-se inferir que o cuidado ético a pessoa estomizada trata-se de um conjunto de postura, atitudes e competências técnicas imprescindíveis para que a assistência de enfermagem seja livre de danos. Além do mais, não se pode fragmentar o cuidado seguro, isto é, olhar apenas para sua faceta técnica, mas urge observar quem desenvolve este cuidado e como este o faz, com seus princípios e valores (MARINS JB, et al., 2018).

É importante compreender o que é cuidado ético, para que possamos saber se os pressupostos do PNSP podem alinhar-se com as necessidades e características das pessoas estomizadas. Vale mencionar que os profissionais de enfermagem e os gestores precisam ter consciência das mudanças na forma de se realizar o cuidado frente aos avanços científicos que presenciamos cotidianamente (BRASIL, 2013).

Por outro lado, cuidado inseguro pode constituir-se um desafio ético pois revelam incertezas, ambiguidades e contradições que, em diversas situações, estão relacionadas a problemas morais, ou seja, desafio que suscita nos profissionais a necessidade de tomar decisão ética, pois em toda ação de cuidado ou de saúde, está permeada por uma ação ética. Os atos de ouvir, discutir, avaliar e dividir opiniões em busca de um consenso, permite desenvolver uma conduta ética necessária para nortear as ações de cuidado e tomada de decisões em saúde (ENDERLE CDF, et al., 2018).

Por fim, quando um dos eixos trata sobre a cultura de segurança que perpassa todos os demais, nesse aspecto estão assegurados um novo pensamento e uma nova postura na produção de saúde, que procura desmitificar e descontruir os antigos conceitos e visões sobre promoção da saúde nos serviços de saúde. Nessa direção, a cultura de segurança contida no PNSP constitui-se um cuidado seguro, e, portanto, ético, pois estimula que as equipes de enfermagem realizem o cuidado sob outra ótica, fundamentado na ciência que permite transformar e ampliar a prática de enfermagem (MAIA MS, 2009; BRASIL, 2013; MARINS JB, 2018).

Portanto, o cuidado seguro inserido no PNSP, constitui-se um cuidado ético, no momento que promove um novo modo de cuidar, de produzir e consumir saúde, pois abarca elementos importantes que estão presentes nos objetivos do Programa. Ao olhar o paciente sob outra perspectiva além da posição passiva que ora ocupa, o PNSP permite ressignificar a prática, estimula a pesquisa e aperfeiçoa as boas práticas baseada em evidências. Incluir os pressupostos do PNSP na formação dos profissionais de saúde, em especial os da enfermagem, como tema transversal, possibilita um cuidado de si e do outro livre de danos e prejuízos quer sejam temporários ou permanentes, respeita com isso a integralidade, individualidade, além de caracterizar-se por um olhar empático para as necessidades das pessoas, que almejam ao adentrarem nos serviços de saúde o seu reestabelecimento (COFEN, 2017; BRASIL, 2013; TRANSFERETTI J, ZACHARIAS R, 2010).

\section{O trabalho em enfermagem e os elementos do Programa de Segurança do Paciente que apontam para um cuidado ético.}

Após a estomização, as pessoas sofrem devido às alterações biopsicossociais, exigindo um cuidado de Enfermagem baseado em conhecimentos e habilidades específicas, capazes de suprir suas necessidades afetadas, além de torná-las capazes de realizar o autocuidado (CENGIZ B e BAHAR Z, 2017). Colaborando para isso, o PNSP aponta elementos importantes que configuram um cuidado ético. Dentre eles, a própria segurança do paciente enquanto atributo da qualidade, composto por elementos como efetividade, 
centralidade no paciente, oportunidade do cuidado, eficiência e equidade. Destes, destaca-se a centralidade no paciente por meio de um cuidado que respeite sua individualidade e responda às suas necessidades de saúde, mantenha seus valores e princípios, bem como sua autonomia, os quais devem direcionar as tomadas de decisões clínicas pelas equipes de saúde assistentes (BRASIL, 2013).

Assim, os elementos do PNSP apontam para um cuidado ético ao promover a reflexão acerca dos benefícios do exercício de um fazer seguro e fundamentado em preceitos e conhecimento científico. Quando as equipes de saúde compreendem esses pressupostos no contexto das pessoas estomizadas, a práxis de enfermagem que se fundamenta na reflexão e alinhamento da teoria com a prática ganha sentido real, pois almeja alcançar o bem-estar e o equilíbrio dessas pessoas diante de suas fragilidades, respeitando seus desejos e produzindo um fazer seguro o qual se se apoia em conhecimento científico (BRASIL, 2013; dos SANTOS BLAN B, MOTA MS, 2020).

No exercício de um cuidado ético, o profissional de enfermagem possibilita que o outro vivencie sentimentos e experimente o não vivido no contexto em que se encontra. Esse processo se dá naturalmente, na presença do profissional disponível. Nesse sentido, a construção de um vínculo por meio de uma escuta qualificada que dê voz aos anseios e preocupações do paciente, possibilita um caminhar em direção de um discurso que transcenda para algo além do que é visto ou falado e, a partir deste avanço, a doença experienciada pelo sujeito poderá despertá-lo para outros movimentos emocionais, desejos e esperança em sua vida (TRANSFERETTI J e ZACHARIAS R, 2010).

Cabe destacar que a enfermagem, de acordo com a Resolução do Conselho Federal de Enfermagem (COFEN) o 564 de 06 de novembro de 2017, é constituída por conhecimentos científicos e técnicos construídos e reproduzidos por um conjunto de práticas sociais, éticas e políticas que resultam em ações de ensino, pesquisa e assistência a qual norteia-se em princípios básicos que são considerados como imperativos para a conduta dos profissionais de enfermagem (COFEN, 2017). Essa Resolução atua como um instrumento orientador para a atuação dos trabalhadores desta categoria, principalmente no que condiz a prática assistencial de qualidade, efetividade e segurança, a fim de evitar situações de negligência, imperícia e imprudência (SILVA TND, et al., 2018).

O cuidado à pessoa com estomia, a partir de um olhar ético, deve considerar as necessidades globais da pessoa cuidada, compreender os aspectos de vida afetados e reconhecer os intensos processos vividos. Ainda, é preciso considerar a presença ou ausência de multimorbidades associadas, a manutenção de relacionamentos e interações sociais, sentimentos positivos e esperançosos, além das facilidades e entraves no acesso ao serviço de saúde e de apoio especializado e apoio familiar (COSTA IKF, et al., 2017).

\section{CONSIDERAÇÕES FINAIS}

Portanto, considera-se que o PNSP possibilita um cuidado ético a partir dos elementos que o compõem. Ao se estabelecer rotinas e protocolos de cuidado seguro, ao exercer o pensamento crítico e construção de uma cultura de segurança pensada no coletivo, estamos cuidando eticamente dos nossos pacientes. Com isso fomenta-se o respeito, pois leva-se em conta as fragilidades e necessidades individuais do ser humano cuidado. Além disso, oportuniza-se a valorização da identidade da pessoa estomizada, por meio da construção de um espaço de voz e de protagonismo no cuidar, na produção de saúde com o mínimo de danos. Possibilita-se a ressignificação de si e do momento vivido, e dos profissionais de saúde envolvidos, pois instiga-se ao comprometimento com a vida na sua singularidade, fundamentado em pressupostos das boas práticas baseadas em evidências. Portanto, esta reflexão pode contribuir para a consolidação do conceito de cuidado ético no exercício da enfermagem, que perceba além das necessidades fisiológicas, mas aponte caminhos para a compreensão das fragilidades do "ser e estar estomizado", pois revela a sua necessidade subjetiva. 


\section{REFERÊNCIAS}

1. ANG SG, et al. Stressors relating to patient psychological health following stoma surgery: an integrated literature review. Oncol Nurs Forum. 2013; 40(6): 587-94.

2. BRASIL. Agência Nacional de Vigilância Sanitária. Implantação do Núcleo de Segurança do Paciente em Serviços de Saúde. Brasília; 2014.

3. BRASIL. Ministério da Saúde. Portaria $\mathrm{n}^{\circ} 529$, de $1^{\circ}$ de abril de 2013. Disponível: http://bvsms.saude.gov.br/bvs/saud elegis/gm/2013/prt0529_01_04_20 13.html. Acesso em: 20 dez. 2020.

4. BRASIL. Portaria $\mathrm{n}$ - 400 , de 16 de novembro de 2009 . Disponível em: http://bvsms.saude.gov.br/bvs/saudelegis/sas/2009/prt0400_16_11_2009.html. Acesso em: 20 dez. 2020.

5. CENGIZ B, BAHAR Z. Perceived Barriers and Home Care Needs When Adapting to a Fecal Ostomy: A Phenomenological Study. J Wound Ostomy Continence Nurs, 2017; 44(1): 63-68.

6. CONSELHO FEDERAL DE ENFERMAGEM. Resolução COFEN no 564/2017. Disponível em: http://www.cofen.gov.br/resolucao-cofen-no-5642017_59145.html. Acesso em: 20 fev. 2021.

7. COSTA IKF, et al. Distúrbio na imagem corporal: diagnóstico de enfermagem e características definidoras em pessoas ostomizadas. Aquichan. 2017; 17(3), 270-283.

8. DA SILVA RA, et al. Assistência de enfermagem ao paciente com estomia intestinal: uma revisão integrativa. Brazilian Journal of Health Review. 2018; 3(4): 10771-10778.

9. DA SILVEIRA RS, et al. A dimensão moral do cuidado em terapia intensiva. Ciência, Cuidado e Saúde. 2014; 13(2), 327-334.

10. DE CARVALHO BARBOSA, CAVALCANTE AK, et al. La atención segura al paciente: contribuciones de enfermería. Revista Cubana de enfermería. 2015; 31(4): 0-0.

11. DOS SANTOS BLAN, B, MOTA, MS, et al. O PROCESSO DE CUIDADO À PESSOA COM ESTOMIA INTESTINAL. Saúde (Santa Maria). 2020; 46(2).

12. ENDERLE CDF, et al. Teaching strategies: promoting the development of moral competence in undergraduate students. Revista Brasileira de Enfermagem. 2018; 71: 1650-1656.

13. GANJALIKHANI MK. Studying the effect of structured ostomy care training on quality of life and anxiety of patients with permanent ostomy. International wound journal. 2019; 16(6): 1383-1390.

14. MACÊDO MS, NOGUEIRA LT, LUZ MHBA. Perfil dos estomizados atendidos em hospital de referência em Teresina. Estima-Brazilian Journal of Enterostomal Therapy. 2005; 3(4).

15. MACIEL DBV. Análise da qualidade de vida em pacientes com estomia intestinal definitiva por câncer. Dissertação (Mestrado em CIÊNCIAS DO CUIDADO EM SAÚDE) - Universidade Federal Fluminense, Niterói, 2018 ; 99 p.

16. MAIA MS. Por uma ética do cuidado. 1 ed. Rio de Janeiro: Garamound; 2009.

17. MARINS JB et al. Formação e aplicabilidade dos preceitos éticos pelos enfermeiros. Enfermagem Brasil. 2018; 17(5): 490-496.

18. MARQUES GS, et al. A vivência de pessoas com estomia intestinal no grupo de apoio em um Hospital Universitário. Revista Hospital Universitário Pedro Ernesto. 2016; 15(2), 113-121.

19. MINAYO MCS. O desafio do conhecimento: pesquisa qualitativa em saúde. 8nd ed. São Paulo: Hucitec, 2013.

20. MOTA MS, GOMES GC, et al. Autocuidado: uma estratégia para a qualidade de vida da pessoa com estomia. Investigación em Enfermería: Imagen y Desarrollo, v. 18, n. 1, p. 63-78, 2016.

21. NÂSVALL P, et al. Quality of life in patients with a permanent stoma after rectal cancer surgery. Qual Life Res. 2017; 26: 55-64.

22. PERES AM, et al. Mapping competencies: identifying gaps in managerial nursing training. Texto \& ContextoEnfermagem. 2017; 26(2).

23. REPIĆ G, et al. Psychological and spiritual well-being aspects of the quality of life in colostomy patients. Vojnosanitetski pregled. 2018; 75(6): 611-617.

24. RIBEIRO WA, et al. As contribuições do enfermeiro no autocuidado ao paciente estomizado. Revista PróUniverSUS. 2019; 10(1): 72-75.

25. SAILER M. Vorbereitung zur Stomaanlage, Patientenedukation und Nachsorge. Coloproctology. 2019; 41(5): 330334.

26. SILVA TND, et al. Vivencia deontológica de la enfermería: develando el código de ética profesional. Revista Brasileira de Enfermagem. 2018; 71(1): 3-10.

27. TRANSFERETTI J, ZACHARIAS R. Ser e cuidar: da ética do cuidado ao cuidado da ética. Aparecida: São Paulo: Editora Santuário; 2010. 